\title{
Nutritional risk, functional status and mortality in newly institutionalised elderly
}

\author{
Emanuele Cereda ${ }^{1 *}$, Carlo Pedrolli ${ }^{2}$, Annunciata Zagami $^{3}$, Alfredo Vanotti ${ }^{4}$, Silvano Piffer ${ }^{5}$, \\ Milena Faliva ${ }^{6}$, Mariangela Rondanelli ${ }^{6}$ and Riccardo Caccialanza ${ }^{1}$ \\ ${ }^{1}$ Nutrition and Dietetics Service, Fondazione IRCCS Policlinico San Matteo, Viale Golgi 19, 27100 Pavia, Italy \\ ${ }^{2}$ Unità Operativa di Dietetica e Nutrizione Clinica, Ospedale "S. Chiara", Azienda Provinciale per i Servizi Sanitari, \\ Trento, Italy \\ ${ }^{3}$ Fondazione Bellaria Onlus, Appiano Gentile, Como, Italy \\ ${ }^{4}$ Servizio di Dietetica e Nutrizione Clinica, ASL Como, Como, Italy \\ ${ }^{5}$ Servizio Osservatorio Epidemiologico, Direzione per la Promozione e l'Educazione alla Salute, \\ Azienda Provinciale per $i$ Servizi Sanitari, Trento, Italy \\ ${ }^{6}$ Servizio Endocrino-nutrizionale, Dipartimento di Scienze Sanitarie Applicate e Psicocomportamentali, \\ Sezione di Nutrizione, Azienda di Servizi alla Persona di Pavia, Università degli Studi di Pavia, Pavia, Italy
}

(Submitted 30 August 2012 - Final revision received 22 February 2013 - Accepted 8 March 2013 - First published online 12 April 2013 )

\section{Abstract}

Previous studies have reported a close relationship between nutritional and functional domains, but evidence in long-term care residents is still limited. We evaluated the relationship between nutritional risk and functional status and the association of these two domains with mortality in newly institutionalised elderly. In the present multi-centric prospective cohort study, involving 346 long-term care resident elderly, nutritional risk and functional status were determined upon admission by the Geriatric Nutritional Risk Index (GNRI) and the Barthel Index (BI), respectively. The prevalence of high (GNRI <92) and low (GNRI 92-98) nutritional risk were 36.1 and 30.6\%, respectively. At multivariable linear regression, functional status was independently associated with age $(P=0 \cdot 045)$, arm muscle area $(P=0 \cdot 048)$, the number of co-morbidities $(P=0.027)$ and mainly with the GNRI $(P<0 \cdot 001)$. During a median follow-up of 4.7 years $(25$ th-75th percentile $3 \cdot 7-6 \cdot 2), 230(66.5 \%)$ subjects died. In the risk analysis, based on the variables collected at baseline, both high (hazard ratio (HR) $1 \cdot 86,95 \%$ CI 1.32, 2.63; $P<0 \cdot 001$ ) and low nutritional risk (HR 1.52, $95 \%$ CI 1.08, 2.14; $P=0 \cdot 016$ ) were associated with all-cause mortality. Participants at high nutritional risk (GNRI <92) also showed an increased rate of cardiovascular mortality (HR 1.93, 95\% CI 1.28, 2.91; $P<0 \cdot 001)$. No association with outcome was found for the BI. Upon admission, nutritional risk was an independent predictor of functional status and mortality in institutionalised elderly. Present data support the concept that the nutritional domain is more relevant than functional status to the outcome of newly institutionalised elderly.

Key words: Nutritional risk (Geriatric Nutritional Risk Index): Functional status (Barthel Index): Long-term care: Elderly: Mortality

The loss of functional status and the related high level of dependence is the main cause of admission to long-term care services $^{(1)}$. Disability may be intrinsic to age, but more frequently reflects other diseases. This is likely to be due to the presence of sarcopenia, which, in turn, has been shown as an independent predictor of outcome ${ }^{(1,2)}$. Malnutrition is a prominent feature of elderly patients admitted to long-term care facilities $^{(3-5)}$. It is associated with adverse outcomes ${ }^{(3-6)}$, and previous studies have found a relationship with functional status ${ }^{(5,7-9)}$. However, the evidence collected in institutions where patients are characterised by the highest levels of dependence is still limited. Moreover, there is no information on the relationship between nutritional risk and functional status. Nutritional screening is currently recommended in any healthcare setting ${ }^{(3,4,10)}$. However, nutritional screening tools do not provide the same information. There is still confusion between an index of nutritional status and that of nutritional risk. The latter takes into account nutritional status-related complications, and it is worthy of mention that a tool's validity should be more consistently discussed in

Abbreviations: BI, Barthel Index; GNRI, Geriatric Nutritional Risk Index; HR, hazard ratio.

*Corresponding author: E. Cereda, fax +39 382 502801, email e.cereda@smatteo.pv.it 
view of the association with outcome. The Geriatric Nutritional Risk Index (GNRI) was proposed to clinicians in 2005 for predicting the risk of nutrition-related complications in a geriatric rehabilitation care setting ${ }^{(11,12)}$, but several studies have supported its use in institutions. The GNRI has been strongly associated with short- and long-term outcomes in this setting and in respect of this, it appears to perform better than the recommended Mini Nutritional Assessment, particularly in newly institutionalised elderly ${ }^{(5,11,13)}$. It has also been associated with the loss of muscle function ${ }^{(14,15)}$ assessed by handgrip strength evaluation. However, the handgrip strength assessment as a surrogate for functional status requires the participation of the patient, which may be frequently unavailable in a long-term care setting. In the present study, we investigated the association between the GNRI and functional status, as assessed by the Barthel Index (BI) of basic activities of daily living, and the relationship of these two domains with mortality in newly institutionalised elderly.

\section{Materials and methods}

\section{Study design}

The present study is part of a larger multi-centric prospective cohort study conducted in four long-term care facilities in the provinces of Como ( $n$ 1), Pavia ( $n$ 1) and Trento $(n 2)$. Baseline data collection began in May 2002 and ended in May 2007. The recruitment procedure was performed as follows: every year, for 2 weeks, all the subjects newly admitted to the facilities, aged $>65$ years and agreeing to participate were assessed for eligibility. Principal exclusion criteria were terminal illness, as assessed by the responsible physician, and a diagnosis of cancer at the time of recruitment ${ }^{(6)}$.

The study was performed in adherence to the principles of the Declaration of Helsinki and the protocol was approved by the local Institutional Ethics Committee. Written informed consent was obtained for every patient.

\section{Nutritional assessment}

Information was collected within $72 \mathrm{~h}$ after admission on the following: body weight; 3-month history of unintentional weight loss (percentage of usual body weight on a continuous scale); height or knee height (for appropriate estimation) ${ }^{(16,17)}$; BMI; arm muscle area (derived from the mid-arm circumference and triceps skinfold thickness) ${ }^{(18)}$. Fasting venous blood samples were assessed for the evaluation of $\mathrm{Hb}$, total lymphocyte count, serum albumin, transthyretin, transferrin, total cholesterol and uric acid. Thereafter, nutritional risk was graded by the GNRI tool. The score was initially derived from the following formula:

$$
\begin{aligned}
\text { GNRI }= & (1.489 \times \text { albumin }(\mathrm{g} / \mathrm{l}))+(41.7 \\
& \times \text { weight } / \text { ideal body weight }) .
\end{aligned}
$$

In this equation, the ideal body weight is calculated from height (either measured or estimated) ${ }^{(17)}$ according to the Lorentz formula and the weight:ideal body weight ratio is set to 1 when the patient's body weight exceeds the ideal body weight ${ }^{(12)}$.

Then, patients were categorised as follows: at high risk, GNRI <92; at low risk, GNRI 92-98; at no risk, GNRI $>98^{(5,16)}$. As was made previously, in the present study, we avoided distinguishing the 'severe risk' group (GNRI <82) from the 'moderate risk' one (GNRI 82-92) because it was found that there is no additional advantage in distinguishing these two categories ${ }^{(8)}$. As the GNRI was designed and validated in $2005^{(12)}$, nutritional risk was retrospectively derived for those patients included before 2005 and prospectively calculated for those entered after its introduction.

\section{Functional status}

Functional status and disability were scored by local trained nurses using the $\mathrm{BI}$, a tool based on questions covering all the aspects of self-care independence in daily living activities (transfer, walking, stairs, toilet use, dressing, feeding, bladder, bowel, grooming and bathing). A total score of 100 points indicates complete self-sufficiency, while a score of zero point means completely dependent ${ }^{(1,19)}$. Given the low median level of functional status and the skewed distribution of data in the present study, participants were categorised into population-specific tertiles (cut-points: 5 and 25) rather than into normal BI score tertiles.

\section{Covariates}

The following information was systematically collected: age; sex; admission diagnosis; all co-morbidities. Diagnoses were coded according to the International Classification of Diseases, 10th Revision. The selection of main admission diagnosis groups to be considered in descriptive and survival analyses was based on the literature review and previous association with nutritional status and/or survival. Data were primarily retrieved from medical records (reported diagnosis) and, in some cases, ascertained through the patient's interview and physical examination. Particularly, hypertension was assessed by the measurement in triplicate of systolic and diastolic blood pressure according to standard procedures, while diabetes was defined by a fasting blood glucose $\geq 1260 \mathrm{mg} / 1$ on two different measurements ${ }^{(20,21)}$. The use of pertinent pharmacological therapy was also taken into account for the assessment of co-morbidities. Finally, data on the use of oral nutritional supplements (sip-feeding) during the stay were also retrieved ${ }^{(6)}$.

\section{Outcome}

Vital status and cause of death were ascertained by active follow-up (Como and Pavia) or by means of record linkage with centralised provincial registry (Trento). Accordingly, events were classified as being due to cardiovascular, respiratory, neoplasms or other/not reported causes by the International Classification of Diseases, 10th Revision. 


\section{Statistical analyses}

Data are presented as means and standard deviations, medians and interquartile ranges (25th-75th percentile) or counts and percentages when appropriate. One-way ANOVA or Kruskal-Wallis test analysis (continuous variables according to a normal and non-normal distribution, respectively) or the $\chi^{2}$ test (categorical variables) were used for the comparison of more than two groups. The independent correlates of functional status were investigated by means of multivariable linear regression analysis. In this model, given the non-normal distribution of data, the BI was entered as a continuous variable on a logarithmic scale. Accordingly, data are reported as $\beta$-coefficients with their standard errors.

Risk factors for mortality were assessed with the Cox proportional hazards regression model and associations are presented as hazard ratios (HR) and 95\% CI. The selection of covariates to be included in the models was initially based on the literature review and previous findings in our study population ${ }^{(6,11,22)}$. With respect to co-morbidities, the sum of all those recorded was used in the analyses. However, in the original cohort ${ }^{(6)}$, diabetes was independently associated with mortality. Accordingly, it has not been included in the total count but has been investigated as an independent predictor of outcome.

In all multivariable analyses, only non-collinear variables (checked by Pearson's statistics) with a $P<0 \cdot 20$ by univariate analysis were included ${ }^{(23)}$.

Statistical analyses were performed using MEDCALC ${ }^{\circledR}$ for Windows version 11.3.0.0 (MedCalc Software) with statistical significance set to a two-tailed $P$ value of $<0.05$.

\section{Results}

From the original cohort ( $n$ 533) assessed during the recruitment phases, 346 patients were included in the present analysis ( $n$ 14, lost to follow-up; $n 173$, incomplete data on the $\mathrm{BI})$. The distribution and the features of the study sample according to risk categories by the GNRI and tertiles of the BI are presented in Table 1. The lack of data on the BI was due to the fact that, in the initial stages of the study, two centres did not collect information on functional status. However, at the group level, features were substantially similar to those of the original cohort in terms of age, sex, anthropometry, biochemistry and the prevalence of main admission diagnoses ${ }^{(6)}$. The population characteristics were also similar among the recruiting centres.

During a median follow-up of 4.7 years ( 25 th -75 th percentile $3 \cdot 7-6 \cdot 2), 230(66 \cdot 5 \%)$ subjects died. The median survival time was significantly reduced in patients at nutritional risk: GNRI $<92,17 \cdot 7$ months (25th-75th percentile 9.4-30.1); GNRI 92-98, 28.8 months (25th-75th percentile 15.4-30.2); GNRI $>98,30 \cdot 8$ months (25th-75th percentile $14 \cdot 2-43.0$ ) ( $P$ for trend $<0.001$; high-risk group significantly different from the others by the post hoc comparison). The survival time was also longer in the case of higher functional status: BI tertile I, 20.5 months (25th-75th percentile 9.3-31.1); BI tertile II, 24.6 months (25th-75th percentile 13.9-30.9);
BI tertile III, $30 \cdot 4$ months (25th-75th percentile $18 \cdot 4-56 \cdot 1)$ ( $P$ for trend $<0.001$; last tertile significantly different from the others by the post hoc comparison). The causes of death were grouped as follows: cardiovascular, $67 \cdot 8 \%$ (coronary artery disease or heart failure $53.2 \%$, stroke $40.4 \%$ and others or unspecified $6.4 \%$ ); respiratory diseases, $25.2 \%$ ( $89.3 \%$ by pneumonia); neoplasm, $2 \cdot 6 \%$; others, $4.4 \%$.

\section{Nutritional risk and functional status}

At multivariable linear regression, functional status by BI (log-transformed) was significantly associated with nutritional risk by GNRI (one-point increase in the score: $\beta=0.018$ (se 0.004); $P<0.001$ ). Other independent correlates were as follows: age (1-year increase: $\beta=-0.007$ (SE 0.004); $P=0.045) ;$ arm muscle area $\left(1 \mathrm{~cm}^{2}\right.$ increase: $\beta=0.009$ (sE 0.004); $P=0.048)$; surrogate for skeletal muscle mass; number of co-morbidities (per additional co-morbidity: $\beta=-0.048$ (se 0.021); $P=0.027$ ).

\section{Nutritional risk, functional status and mortality}

In multivariable analysis, all-cause mortality (Table 2; Fig. 1) was significantly associated with nutritional risk. Other independent predictors were age, sex, number of co-morbidities and diabetes. However, the association between functional status and outcome was significant only in univariate analysis. Due to a slight collinearity (Pearson's $r$ 0.31) between the GNRI and the BI, we also tested the possible interaction $($ GNRI $\times$ BI) between these two domains in outcome prediction. The association was not significant $(P=0 \cdot 068)$.

Similar results were found in cause-specific analysis where nutritional risk was the main predictor of cardiovascular mortality (model adjusted also for hypertension): GNRI $<92 \mathrm{HR}$ 1.93 (95\% CI 1.28, 2.91), $P<0.001$; GNRI 92-98 HR 1.31 (95\% CI $0 \cdot 85,1.99), P=0 \cdot 221$ (linear increase over risk categories, HR $0 \cdot 71,95 \%$ CI $0.58,0 \cdot 88, P=0.001)$; BI HR 0.95 (95\% CI $0.78,1 \cdot 16$ ), $P=0.619$ (linear increase over tertiles). Other causes were not investigated due to the limited number of events.

During the follow-up, sixty-nine participants (19.9\%) received oral nutritional supplements (sip-feeding). The frequency of nutritional support among the GNRI categories and BI tertiles was GNRI <92, 16.8\%; GNRI 92-98, 28.3\%; GNRI $>98,15 \cdot 7 \%$; BI tertile I, $19 \cdot 0 \%$; BI tertile II, $25 \cdot 2 \%$; BI tertile III, $15 \cdot 6 \%$. Risk analyses adjusted for the use of oral nutritional supplements confirmed the associations found. Nutritional support was also associated with reduced mortality risk (all-cause: HR 0.58 (95\% CI 0.34, 0.98), $P=0.045$; cardiovascular: HR 0.54 (95\% CI 0.32, 1.00), $P=0 \cdot 050$ ).

Finally, fully adjusted risk models (sample size, $n$ 259) refitted after the exclusion of participants who died in the first year of follow-up confirmed all the associations found with both all-cause (events, $n$ 143; GNRI <92 HR 1.98 (95\% CI $1 \cdot 27,3 \cdot 09), P=0 \cdot 003)$; GNRI 92-98 HR 1.81 (95\% CI $1 \cdot 19$, 2.75), $P=0.006$ ) and cardiovascular (events, $n$ 95; GNRI $<92$ HR 2.14 (95\% CI 1.26, 3.66), $P=0.005$ ); GNRI 92-98 HR 1.59 (95\% CI 0.94, 2.67), $P=0.083)$ mortality. 
Table 1. Baseline clinical and demographic characteristics of the population by Geriatric Nutritional Risk Index (GNRI) and Barthel Index (BI) categories (Mean values and standard deviations; medians and interquartile ranges (IQR); percentages)

\begin{tabular}{|c|c|c|c|c|c|c|c|c|c|c|c|c|c|c|c|c|}
\hline \multirow[b]{3}{*}{ Characteristics $†$} & \multirow{2}{*}{\multicolumn{2}{|c|}{$\begin{array}{l}\text { Overall } \\
\text { population } \\
(n 346)\end{array}$}} & \multicolumn{2}{|c|}{ High risk } & \multicolumn{2}{|c|}{ Low risk } & \multicolumn{2}{|c|}{ No risk } & \multirow[b]{3}{*}{$P \neq$} & \multicolumn{2}{|c|}{ First tertile } & \multicolumn{2}{|c|}{ Second tertile } & \multicolumn{2}{|c|}{ Third tertile } & \multirow[b]{3}{*}{$P \ddagger$} \\
\hline & & & \multicolumn{2}{|c|}{$\begin{array}{c}\text { GNRI }<92 \\
(n 125)\end{array}$} & \multicolumn{2}{|c|}{$\begin{array}{l}\text { GNRI 92-98 } \\
\quad(n \text { 106) }\end{array}$} & \multicolumn{2}{|c|}{$\begin{array}{c}\text { GNRI }>98 \\
(n 115)\end{array}$} & & \multicolumn{2}{|c|}{$\begin{array}{l}\text { BI } 0-5 \\
(n 116)\end{array}$} & \multicolumn{2}{|c|}{$\begin{array}{l}\text { BI 6-25 } \\
(n 115)\end{array}$} & \multicolumn{2}{|c|}{$\begin{array}{l}\mathrm{BI}>25 \\
(n 115)\end{array}$} & \\
\hline & Mean & SD & Mean & SD & Mean & SD & Mean & SD & & Mean & SD & Mean & SD & Mean & SD & \\
\hline Male (\%) & \multicolumn{2}{|c|}{25.4} & \multicolumn{2}{|c|}{$20 \cdot 0$} & \multicolumn{2}{|c|}{$22 \cdot 6$} & \multicolumn{2}{|c|}{30.4} & 0.152 & \multicolumn{2}{|c|}{$25 \cdot 9$} & \multicolumn{2}{|c|}{$18 \cdot 3$} & \multicolumn{2}{|c|}{$28 \cdot 7$} & 0.162 \\
\hline Age (years) & 85.7 & $9 \cdot 1$ & 86.7 & $10 \cdot 9$ & 85.4 & 7.5 & $85 \cdot 0$ & $8 \cdot 1$ & 0.284 & 86.9 & 7.5 & $86 \cdot 1$ & 10.4 & $84 \cdot 1^{*}$ & 8.9 & 0.053 \\
\hline GNRI & 93.9 & $7 \cdot 8$ & $85 \cdot 4^{\star}$ & $5 \cdot 2$ & $94 \cdot 7^{*}$ & 1.8 & $102 \cdot 5^{\star}$ & 3.9 & $<0.001$ & 91.4 & 8.6 & 93.1 & 8.0 & $97 \cdot 3^{*}$ & $6 \cdot 8$ & $<0.001$ \\
\hline $\mathrm{BI}$ & & & & & & & & & $<0.001$ & & & & & & & $<0.001$ \\
\hline Median & & & & & & & & & & & & & & & & \\
\hline IQR & & & & & & & & & & & & & & & & \\
\hline $\mathrm{BMI}\left(\mathrm{kg} / \mathrm{m}^{2}\right)$ & $23 \cdot 1$ & 4.8 & $20 \cdot 8^{\star}$ & 4.4 & $24 \cdot 2$ & 4.8 & 24.5 & 4.3 & $<0.001$ & $22 \cdot 3$ & 4.5 & $22 \cdot 1$ & 4.8 & $24 \cdot 7^{*}$ & 4.7 & $<0.001$ \\
\hline$<18.5$ & & & & & & & & & $<0.001$ & & & & & & & $<0.001$ \\
\hline $18 \cdot 5-25$ & & & & & & & & & & & & & & & & \\
\hline $25-30$ & & & & & & & & & & & & & & & & \\
\hline$\geq 30$ & & & & & & & & & & & & & & & & \\
\hline Arm muscle area $\left(\mathrm{cm}^{2}\right)$ & 34.8 & $10 \cdot 6$ & $30.9^{*}$ & $8 \cdot 7$ & 35.9 & 9.9 & $37 \cdot 7$ & 11.8 & $<0.001$ & 34.4 & 11.3 & 33.8 & $10 \cdot 1$ & $36 \cdot 2$ & $10 \cdot 4$ & 0.208 \\
\hline Weight loss (\%) & -0.5 & 3.0 & -2.4 & 3.7 & $0 \cdot 2^{\star}$ & $4 \cdot 1$ & -0.3 & $1 \cdot 8$ & 0.028 & -0.8 & 3.4 & -1.3 & $4 \cdot 2$ & -0.1 & $2 \cdot 4$ & 0.349 \\
\hline Albumin $(g / l)$ & $36 \cdot 8$ & 4.8 & $32 \cdot 6^{*}$ & 3.4 & $36 \cdot 7^{\star}$ & $2 \cdot 11$ & $41 \cdot 5^{\star}$ & $2 \cdot 7$ & $<0.001$ & 35.5 & $5 \cdot 0$ & $36 \cdot 7$ & 4.4 & $38 \cdot 1^{*}$ & $4 \cdot 2$ & $<0.001$ \\
\hline Transthyretin (mg/l) & 189 & 64 & $144^{\star}$ & 55 & 197 & 63 & 216 & 54 & $<0.001$ & 172 & 58 & 184 & 62 & $203^{*}$ & 67 & 0.014 \\
\hline Transferrin (mg/l) & 1950 & 400 & $1770^{*}$ & 380 & 1960 & 480 & 2080 & 340 & $<0.001$ & $1810^{*}$ & 440 & 2000 & 420 & 2030 & 370 & 0.001 \\
\hline $\mathrm{Hb}(\mathrm{g} / \mathrm{l})$ & 117 & 29 & 116 & 29 & 112 & 30 & 122 & 27 & 0.174 & 121 & 27 & 115 & 29 & 116 & 30 & 0.672 \\
\hline Lymphocyte count (per $\mathrm{mm}^{3}$ ) & 1837 & 687 & 1784 & 782 & 1838 & 591 & 1893 & 663 & 0.508 & 1806 & 764 & 1762 & 578 & 1948 & 696 & 0.132 \\
\hline Total cholesterol (mg/l) & 1730 & 360 & 1620 & 360 & 1740 & 300 & $1900^{*}$ & 360 & $<0.001$ & 1690 & 360 & 1740 & 420 & 1730 & 270 & 0.376 \\
\hline Uric acid (mg/l) & 53 & 17 & 51 & 17 & 51 & 17 & 60 & 18 & 0.004 & $56^{*}$ & 18 & 52 & 17 & 53 & 16 & 0.269 \\
\hline Main admission diagnoses (\%) & & & & & & & & & & & & & & & & \\
\hline All-type dementia & & & & & & & & & 0.602 & & & & & & & 0.533 \\
\hline Heart disease & & & & & & & & & 0.898 & & & & & & & 0.010 \\
\hline Hip fracture & & & & & & & & & 0.002 & & & & & & & 0.499 \\
\hline Psychiatric disorder & & & & & & & & & 0.053 & & & & & & & 0.007 \\
\hline Stroke & & & & & & & & & 0.796 & & & & & & & 0.378 \\
\hline Others & & & & & & & & & 0.809 & & & & & & & 0.809 \\
\hline Co-morbidities & $2 \cdot 7$ & 1.5 & $3 \cdot 0^{*}$ & 1.6 & $2 \cdot 8$ & 1.4 & $2 \cdot 4$ & 1.5 & 0.012 & $2 \cdot 9$ & 1.5 & $2 \cdot 9$ & 1.5 & $2 \cdot 4^{*}$ & 1.5 & 0.010 \\
\hline Main co-morbidities (\%) & & & & & & & & & & & & & & & & \\
\hline Diabetes & & & & & & & & & 0.098 & & & & & & & 0.866 \\
\hline Hypertension & & & & & & & & & 0.369 & & & & & & & 0.093 \\
\hline Pressure ulcer(s) & & & & & & & & & 0.005 & & & & & & & 0.085 \\
\hline
\end{tabular}

*Values were significantly different between the groups $(P<0.05$; post hoc test).

† Percentages are calculated within the single groups.

$\ddagger$ Continuous and categorical variables were compared between the groups with one-way ANOVA or the $\chi^{2}$ test, respectively. 
Table 2. Predictors of all-cause mortality according to Cox proportional hazards regression models

(Hazard ratios and $95 \%$ confidence intervals)

\begin{tabular}{|c|c|c|c|c|c|c|}
\hline \multirow[b]{2}{*}{ Characteristics } & \multicolumn{6}{|c|}{ All-cause mortality } \\
\hline & Hazard ratio* & $95 \% \mathrm{Cl}$ & $P$ & Hazard ratio† & $95 \% \mathrm{Cl}$ & $P$ \\
\hline \multicolumn{7}{|l|}{ Sex } \\
\hline Male & 1.73 & $1.31,2 \cdot 30$ & $<0.001$ & 2.03 & $1 \cdot 52,2 \cdot 72$ & $<0.001$ \\
\hline Age (years) & 1.04 & $1.03,1.06$ & $<0.001$ & 1.05 & $1.03,1.07$ & $<0.001$ \\
\hline GNRI categories & $0.74 \ddagger$ & $0.63,0.87$ & $<0.001 \ddagger$ & $0.74 \ddagger$ & $0.62,0.87$ & $<0.001 \ddagger$ \\
\hline$<92$ & 1.84 & $1.33,2.53$ & $<0.001$ & 1.86 & $1.32,2.63$ & $<0.001$ \\
\hline $92-98$ & 1.46 & $1.05,2.02$ & 0.026 & 1.52 & $1 \cdot 08,2 \cdot 14$ & 0.016 \\
\hline$>98$ & 1 & Reference & & 1 & Reference & \\
\hline Barthel Index tertiles & $0.85 \ddagger$ & $0.76,0.99$ & $0.043 \ddagger$ & $0.96 \ddagger$ & $0.82,1 \cdot 13$ & $0.658 \ddagger$ \\
\hline \multicolumn{7}{|c|}{ Main admission diagnoses } \\
\hline Hip fracture & 1.34 & $0.87,2.08$ & 0.188 & 1.25 & $0.79,1.97$ & 0.334 \\
\hline Psychiatric disorder & 0.67 & $0.40,1 \cdot 11$ & 0.120 & 0.84 & $0.49,1.42$ & 0.513 \\
\hline Diabetes & 1.46 & $1.05,2.04$ & 0.026 & 1.53 & $1.02,2.29$ & 0.039 \\
\hline Co-morbidities§ & $1 \cdot 13$ & $1.03,1.23$ & 0.007 & $1 \cdot 13$ & $1.01,1.29$ & 0.032 \\
\hline
\end{tabular}

GNRI, Geriatric Nutritional Risk Index.

${ }^{*}$ Risk of death at univariate analysis (variables presented were those having a $P$ value $<0.20$ )

† Risk of death at multivariable analysis.

‡Linear increase in risk over the categories assumed (checked with the likelihood ratio test).

$\S$ Per additional co-morbidity (other than diabetes).

\section{Discussion}

In the present study, it was found that nutritional risk, as assessed by the GNRI, is significantly associated with functional status and outcomes in newly institutionalised elderly. However, no association between functional status and mortality was observed.

The present results, which support the association between nutritional risk by the GNRI and mortality, are in agreement with the observations recently collected in a similar cohort $^{(11,13)}$ and with those preliminarily provided by some of the present investigators ${ }^{(24)}$. However, compared with the findings reported few years ago, both high and low nutritional risks have been associated with poor prognosis. This difference could be reasonably ascribed to the greater sample size and the characteristics of the patients included (only newly admitted).

A limited number of studies have focused on the association between nutritional and functional status in a long-term care setting $^{(7,9,11,14,25)}$. Moreover, the level of available evidence is hampered by the heterogeneity of the tools used for the assessment of both nutritional and functional domains, by the various underlying diseases and by the different sample sizes investigated. Moreover, there is still confusion between an index of nutritional status and that of nutritional risk, because the latter is more likely to account for nutritional status-related complications. Although several tools for nutritional screening are now available, recent literature supports the value of the GNRI in this patient population, due to its relationship with several parameters of nutritional status, muscle function and outcomes ${ }^{(5)}$. Moreover, the prognostic value in newly institutionalised elderly appears superior to that of the recommended Mini Nutritional Assessment ${ }^{(5,8,11)}$. Having a tool such as the GNRI in the evaluation of the patient may be an advantage because it requires minimal participation of the patient and, at the same time, it is able to both reflect functional status and predict the outcome. The strong relationship between the GNRI and the BI presented herein expands previous knowledge that was mainly related to disability as assessed by handgrip strength ${ }^{(14,15)}$. Indeed, there is supportive evidence that the prevalence of nutritional derangements is inversely associated with the setting-related level of dependence, which, in turn, relies on functional status $^{(2,8)}$. In a previous investigation, we have reported that the association between nutritional status and the BI was substantially explained by weight loss, muscle mass and energy intake $^{(7)}$. In the present study, we did not assess energy intake, a factor that seems to improve the identification of muscle dysfunction independently of nutritional risk ${ }^{(14)}$. We have previously discussed that the prognostic value of the GNRI lies in its structure, which is mainly based on serum albumin as well as on the deviation of body weight from ideal conditions. As a nutritional marker, serum albumin is likely to account for disease severity and acute changes in nutritional balance ${ }^{(5,26)}$. Looking at the GNRI structure, we could say that also the body weight factor is of help in describing the status and turnover of protein stores ${ }^{(5,26)}$. Nutritional derangements are related to the underlying disease ${ }^{(25)}$, which is recognised as an important determinant of functional status ${ }^{(1)}$. However, the association between the GNRI and the BI was independent of admission diagnosis. Accordingly, it could be sustained that the GNRI is likely to account for functional and metabolically active components of the body, which, in turn, have been associated with outcome ${ }^{(5,9,27)}$. However, the cause-effect relationship behind the association between nutritional and functional status is difficult to establish; however, recent data support the concept of functionality being more relevant for nutritional status ${ }^{(9)}$. This issue should be the object of prospective investigations. In the present study, functional status was not an independent predictor of mortality in multivariable analysis. The result may appear in contrast with previous findings ${ }^{(28)}$. However, it should be highlighted that most of the evidence on the association with adverse outcomes has 

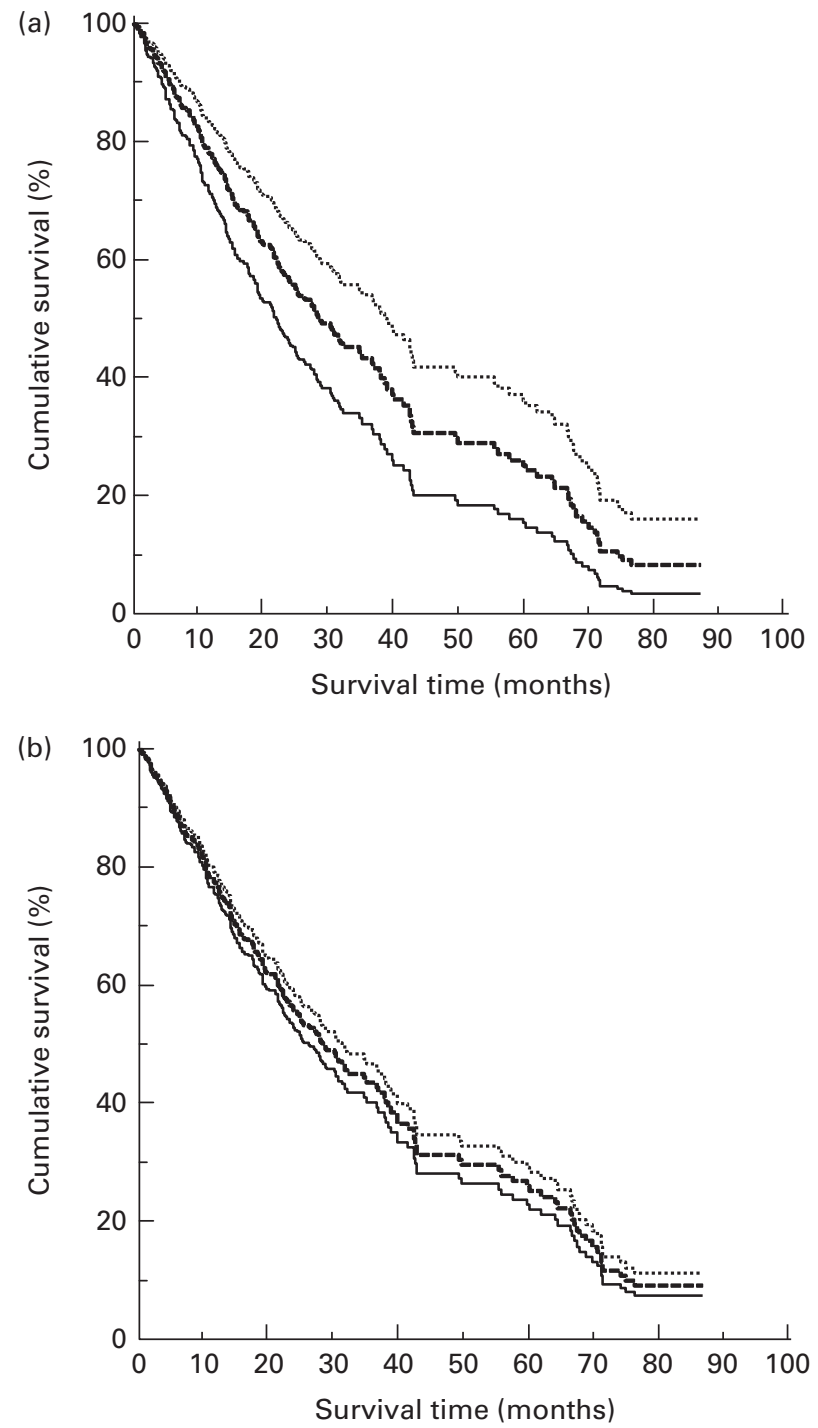

Fig. 1. Adjusted cumulative survival curves for all-cause mortality across (a) nutritional risk (Geriatric Nutritional Risk Index) categories $(P<0.001 ;$ high risk; ---..., low risk; ........., no risk) and (b) tertiles of the Barthel Index ( $P=0.658 ; \ldots$, first tertile; ----.., second tertile; .........., third tertile).

been collected in community-living elderly ${ }^{(28)}$, in particular sets of patients (e.g. stroke) ${ }^{(29)}$, or using data recorded in acute or post-acute conditions ${ }^{(30-32)}$. Accordingly, patients could be characterised by more heterogeneous conditions, while in the present study, about $70 \%$ of the population had a BI score $<30$ points, thus suggesting that in the presence of high levels of dependence, other factors are more likely to affect the outcomes. Nonetheless, the recent study by Kaiser et al. ${ }^{(9)}$ supports the evidence that functional status is less relevant to outcomes even in the presence of lower levels of dependence.

Finally, in agreement with previous observations ${ }^{(11)}$, this was an interesting finding that nutritional risk is associated with cardiovascular mortality. It could be speculated that changes in inflammatory and hormonal status (glucocorticoids and catecholamines) and the dysregulation of the autonomic nervous system occurring with malnutrition and ageing both act as risk modifiers ${ }^{(33-35)}$.
Some other limitations should be considered. First, the low level of dependence and the choice to categorise participants on the basis of BI data distribution make the results strictly linked to the sample population, hampering their generalisability and the comparison with other data in the literature. The same may also be argued for the prevalence of some major co-morbidities, such as diabetes and hypertension. Second, a consistent number of patients (approximately $30 \%$ ) of the original cohort have been excluded due to the lack of BI data. However, we believe that this did not represent a source of bias because it was observed that the present study population was comparable with the original cohort and features were similar among the recruiting centres. Third, the findings could have been strengthened by the evaluation of inflammatory background, although the association between nutritional risk and functional status was independent of co-morbidities. Fourth, as frequently occurs in observational studies, the effect of several potential factors acting during the follow-up has not been taken into account, particularly the main outcome predictors have been assessed only upon admission. Accordingly, it could not be excluded that changes in functional status occurring during the follow-up could play a role in modifying the patient's outcome. On the other hand, albumin, being a marker of disease severity, is more likely to be associated with outcome. Fifth, the cognitive capacity of those people without a diagnosis of dementia has not been fully explored by the nursing home staff and residual bias in the association between functional and nutritional status may have occurred. However, the association between these two domains was the strongest in our multivariable model, and it is reasonable to argue that the results were affected only to a minimal extent. Finally, as previously discussed, the introduction of risk estimation bias could not be excluded due to the procedure of recruitment through which patients surviving complications leading to institutionalisation were more likely to be included.

In summary, nutritional risk is significantly associated with functional status and mortality (all-cause and cardiovascular) in newly institutionalised elderly. The present study supports the concept that the nutritional domain is more relevant than functional status to the outcome of newly institutionalised elderly. The potential role of nutritional support in improving the outcome is also suggested.

\section{Acknowledgements}

The study was supported by the Fondazione IRCCS Policlinico San Matteo, Pavia, Italy. E. C. received consultancy honoraria and investigator grants from Nutricia Italia and the 'Fondazione Grigioni per il Morbo di Parkinson'. Abstract accepted as oral presentation and awarded a 'Travel Fellowship' at the upcoming 34th ESPEN Congress 2012, Barcelona, Spain.

All authors significantly contributed to the study, read and approved the final version of the manuscript. E. C. had full access to all of the data in the study and took responsibility for the integrity of the data and the accuracy of the data analysis. E. C., C. P. and A. V. contributed to the study concept and design. E. C., C. P., S. P., M. F., M. R. and A. Z. were involved in 
the acquisition of the data. E. C. and C. P. participated in the analysis and the interpretation of the data. E. C. drafted the manuscript. E. C., R. C., C. P. and M. R. critically revised the manuscript for important intellectual content.

The authors certify that there are no affiliations with or involvement in any organisation or entity with a direct financial interest in the subject matter or materials discussed in the manuscript.

\section{References}

1. Quinn TJ, McArthur K, Ellis G, et al. (2011) Functional assessment in older people. BMJ 343, d4681.

2. Cruz-Jentoft AJ, Baeyens JP, Bauer JM, et al. (2010) Sarcopenia: European consensus on definition and diagnosis: Report of the European Working Group on Sarcopenia in Older People. Age Ageing 39, 412-423.

3. Salva A, Coll-Planas L, Bruce S, et al. (2009) Nutritional assessment of residents in long-term care facilities (LTCFs): recommendations of the task force on nutrition and ageing of the IAGG European region and the IANA. $J$ Nutr Health Aging 13, 475-483.

4. Arvanitakis M, Beck A, Coppens P, et al. (2008) Nutrition in care homes and home care: how to implement adequate strategies (report of the Brussels Forum (22-23 November 2007)). Clin Nutr 27, 481-488.

5. Cereda E \& Pedrolli C (2009) The geriatric nutritional risk index. Curr Opin Clin Nutr Metab Care 12, 1-7 (erratum in: Curr Opin Clin Nutr Metab Care 12, 683).

6. Cereda E, Pedrolli C, Zagami A, et al. (2011) Body mass index and mortality in institutionalized elderly. J Am Med Dir Assoc 12, 174-178.

7. Cereda E, Valzolgher L \& Pedrolli C (2008) Mini nutritional assessment is a good predictor of functional status in institutionalised elderly at risk of malnutrition. Clin Nutr 27, $700-705$.

8. Cereda E (2012) Mini nutritional assessment. Curr Opin Clin Nutr Metab Care 15, 29-41.

9. Kaiser R, Winning K, Uter W, et al. (2010) Functionality and mortality in obese nursing home residents: an example of 'risk factor paradox'? J Am Med Dir Assoc 11, 428-435.

10. Kondrup J, Allison SP, Elia M, et al. (2003) ESPEN guidelines for nutrition screening 2002. Clin Nutr 22, 415-421.

11. Cereda E, Pedrolli C, Zagami A, et al. (2011) Nutritional screening and mortality in newly institutionalised elderly: a comparison between the geriatric nutritional risk index and the mini nutritional assessment. Clin Nutr 30, 793-798.

12. Bouillanne O, Morineau G, Dupont C, et al. (2005) Geriatric nutritional risk index: a new index for evaluating at-risk elderly medical patients. Am J Clin Nutr 82, 777-783.

13. Cereda E, Pusani C, Limonta D, et al. (2009) The ability of the geriatric nutritional risk index to assess the nutritional status and predict the outcome of homecare resident elderly: a comparison with the mini nutritional assessment. Br J Nutr 102, 563-570.

14. Cereda E \& Vanotti A (2008) Short dietary assessment improves muscle dysfunction identification by geriatric nutritional risk index in uncomplicated institutionalised patients over 70 years old. Clin Nutr 27, 126-132.

15. Cereda E \& Vanotti A (2007) The new geriatric nutritional risk index is a good predictor of muscle dysfunction in institutionalized older patients. Clin Nutr 26, 78-83.

16. Cereda E, Bertoli S, Vanotti A, et al. (2010) Estimated height from knee-height in Caucasian elderly: implications on nutritional status by mini nutritional assessment. $J$ Nutr Health Aging 14, 16-22.

17. Cereda E, Limonta D, Pusani C, et al. (2007) Feasible use of estimated height for predicting outcome by the geriatric nutritional risk index in long-term care resident elderly. Gerontology 53, 184-186.

18. World Health Organization (1995) Physical status: the use and interpretation of anthropometry. Report of a WHO Expert Committee. World Health Organ Tech Rep Ser 854, $1-452$.

19. Mahoney FI \& Barthel DW (1965) Functional evaluation: the Barthel index. Med State Med J 14, 61-65.

20. Chobanian AV, Bakris GL, Black HR, et al. (2003) Seventh report of the Joint National Committee on Prevention, Detection, Evaluation, and Treatment of High Blood Pressure. Hypertension 42, 1206-1252.

21. American Diabetes Association (2006) Diagnosis and classification of diabetes mellitus. Diabetes Care 29, Suppl. 1, S43-S48.

22. Cereda E, Pedrolli C, Zagami A, et al. (2013) Alzheimer's disease and mortality in traditional long-term care facilities. Arch Gerontol Geriatr 56, 437-441.

23. Haynes RB, Sackett DL, Guyatt GH, et al. (2006) Clinical Epidemiology: How to Do Clinical Practice Research, 3rd ed. Philadelphia, PA: Lippincott Williams \& Wilkins.

24. Cereda E, Zagami A, Vanotti A, et al. (2008) Geriatric nutritional risk index and overall-cause mortality prediction in institutionalised elderly: a 3-year survival analysis. Clin Nutr 27, 717-723.

25. Bahat G, Saka B, Tufan F, et al. (2010) Prevalence of sarcopenia and its association with functional and nutritional status among male residents in a nursing home in Turkey. Aging Male 13, 211-214.

26. Ballmer PE (2001) Causes and mechanisms of hypoalbuminaemia. Clin Nutr 20, 271e3.

27. Volpato S, Romagnoni F, Soattin L, et al. (2004) Body mass index, body cell mass, and 4-year all-cause mortality risk in older nursing home residents. J Am Geriatr Soc 52, 886-891.

28. Cooper R, Kuh D, Hardy R, et al. (2010) Objectively measured physical capability levels and mortality: systematic review and meta-analysis. BMJ 341, c4467.

29. Huybrechts KF \& Caro JJ (2007) The Barthel index and modified Rankin scale as prognostic tools for long-term outcomes after stroke: a qualitative review of the literature. Curr Med Res Opin 23, 1627-1636.

30. Bellelli G, Magnifico F \& Trabucchi M (2008) Outcomes at 12 months in a population of elderly patients discharged from a rehabilitation unit. J Am Med Dir Assoc 9, 55-64.

31. Espaulella J, Arnau A, Cubí D, et al. (2007) Time-dependent prognostic factors of 6-month mortality in frail elderly patients admitted to post-acute care. Age Ageing 36, 407-413

32. Baztán JJ, Gálvez CP \& Socorro A (2009) Recovery of functional impairment after acute illness and mortality: oneyear follow-up study. Gerontology 55, 269-274.

33. Avraham Y, Hao S, Mendelson S, et al. (2002) Hypothalamic-pituitary-adrenal responses to weight loss in mice following diet restriction, activity or separation stress: effects of tyrosine. Nutr Neurosci 5, 327-335.

34. Venihaki M, Dikkes P, Carrigan A, et al. (2001) Corticotropinreleasing hormone regulates IL-6 expression during inflammation. J Clin Invest 108, 1159-1166.

35. Yeh SS \& Schuster MW (1999) Geriatric cachexia: the role of cytokines. Am J Clin Nutr 70, 183-197. 